Stockholm

August 2008

Revised October 2008

\title{
THE FRAME POTENTIAL, ON AVERAGE
}

\author{
Ingemar Bengtsson ${ }^{1}$ \\ Stockholm University, AlbaNova \\ Fysikum \\ S-106 91 Stockholm, Sweden \\ Helena Granström² \\ Stockholm University \\ Institutionen för matematik \\ S-106 91 Stockholm, Sweden
}

\begin{abstract}
A SIC consists of $N^{2}$ equiangular unit vectors in an $N$ dimensional Hilbert space. The frame potential is a function of $N^{2}$ unit vectors. It has a unique global minimum if the vectors form a SIC, and this property has been made use of in numerical searches for SICs. When the vectors form an orbit of the Heisenberg group the frame potential becomes a function of a single fiducial vector. We analytically compute the average of this function over Hilbert space. We also compute averages when the fiducial vector is placed in certain special subspaces defined by the Clifford group.
\end{abstract}

\footnotetext{
${ }^{1}$ Email address: ingemar@physto.se. Supported by VR.

${ }^{2}$ Email address: helenag@math.su.se.
} 


\section{Introduction}

Symmetric Informationally Complete Positive Operator Valued Measures, or SICs, is the unwieldy name for a simple idea [1,2]: a set of $N^{2}$ unit vectors in an $N$ dimensional Hilbert space, equiangular in the sense that

$$
\left|\left\langle\psi_{I} \mid \psi_{J}\right\rangle\right|^{2}=\frac{1}{N+1}, \quad 1 \leq I, J \leq N, \quad I \neq J .
$$

If the vectors are reinterpreted as projectors, that is as points in the set of Hermitian matrices of unit trace (the space where the density matrices live), they form a regular simplex in an $N^{2}-1$ dimensional Euclidean space. This also explains why we want their number to be $N^{2}$. The corners of such a simplex can be used to define barycentric coordinates for any density matrix, which is what "informationally complete" stands for. In quantum information theory SICs have attracted attention because they - if they exist - are useful for quantum state tomography $[2,3]$. In quantum foundations they have attracted attention as a preferred kind of measurement [4] - and they have been studied in many other branches of science under names such as "equiangular lines" [5], "equiangular tight frames" [6], and "maximal quantum designs" [1]. Strohmer and Heath provide a mathematical survey [7].

The question whether SICs exist for all $N$ has turned out to be extraordinarily difficult to answer. They have been constructed in most (but not all) dimensions $N \leq 19[1,8,9,10,11]$. Numerical searches have been successful for all $N \leq 45$ [2], but no general formula has emerged. This is a bit surprising, given that we are really asking a very simple question about the shape of the convex body of density matrices: is it possible to inscribe a regular simplex in this body, with $N^{2}$ corners on its outsphere? The available evidence does however suggest that the answer is "yes" for all $N$, and moreover one can always find a SIC covariant under the Heisenberg-Weyl group, meaning that it can be constructed by first choosing a fiducial vector $\left|\psi_{0}\right\rangle$, and then acting on this vector with the $N^{2}$ elements of the (projective) Heisenberg-Weyl group.

A natural measure of how close a given set of $N^{2}$ unit vectors $\left|\psi_{I}\right\rangle$ is to forming a SIC is given by the function

$$
f \equiv \frac{1}{2} \sum_{I \neq J}\left(\left|\left\langle\psi_{I} \mid \psi_{J}\right\rangle\right|^{2}-\frac{1}{N+1}\right)^{2} .
$$


By construction $f=0$ if and only if the vectors form a SIC. The function $f$ is related to the frame potentials

$$
F_{t} \equiv \sum_{I, J}\left|\left\langle\psi_{I} \mid \psi_{J}\right\rangle\right|^{2 t}, \quad t \in\{1,2\}
$$

by

$$
f=\frac{1}{2} F_{2}-\frac{1}{N+1} F_{1} .
$$

These frame potentials also assume their global minimum for a SIC, and in fact it is enough if $F_{2}$ assumes its minimum, since $F_{1}$ is known to follow suit. Therefore numerical searches for SICs have focused on minimizing $F_{2}$. Actually a frame potential is defined for any integer $t[2,6]$, but this does not concern us here.

We will have nothing to say about the existence problem here, rather we will compute averages of the function $f$, with and without the assumption of group covariance. We use the Fubini-Study measure on complex projective space to perform the averaging. We also compute averages when the fiducial vector is restricted to lie in certain subspaces defined by the Heisenberg group and its normalizer (the so-called Clifford group). These calculations were made for a reason: in the course of an investigation of certain configurations of $N^{2}$ vectors that occur in a related problem (to be precise but perhaps not informative, the torsion points of an elliptic curve defined by a complete set of mutually unbiased bases) we computed the values of $f$ for these configurations. And the question then arose whether the values we obtained were high or low. To answer questions like this - and there are many such questions - one needs to know these averages.

Our final results have a certain elegance. Our paper is organized as follows: in section 2 we state some definitions, and in section 3 we describe the special subspaces we are interested in. In section 4 we give details concerning our calculations, which were carried out by brute force. Readers who are familiar with the SIC problem, and who do not want the details of an involved calculation, are advised to go directly to section 5 , where we state our results and make some comments. ${ }^{3}$

\footnotetext{
${ }^{3}$ The first version of this paper contained a mistake, in eq. (34). A counterintuitive and exciting result ensued. The mistake was spotted by Christopher Fuchs and by an anonymous referee; it is better to be right than to be exciting, and we thank them for this.
} 


\section{Definitions}

The Heisenberg-Weyl group [12] is the group generated by two elements $\tau$ and $\sigma$ subject to the relations

$$
\sigma \tau=q \tau \sigma, \quad \tau^{N}=\sigma^{N}=1, \quad q \equiv e^{2 \pi i / N} .
$$

Up to unitary equivalence there is a unique unitary representation of this group such that

$$
\sigma|a\rangle=q^{a}|a\rangle \quad \tau|a\rangle=|a+1\rangle, \quad 0 \leq a \leq N-1 .
$$

This is how we fix coordinates in Hilbert space; vectors can then be defined by their components

$$
|\psi\rangle=\sum_{a=0}^{N-1} Z^{a}|a\rangle .
$$

Up to phases, a general group element is

$$
D_{i j}=\tau^{i} \sigma^{j}, \quad 0 \leq i, j \leq N-1 .
$$

The phases do not matter to us.

Now consider $N^{2}$ unit vectors forming an orbit under the Heisenberg group,

$$
\left|\psi_{i j}\right\rangle=D_{i j}\left|\psi_{0}\right\rangle
$$

where $\left|\psi_{0}\right\rangle$ is some fiducial unit vector. The frame potential evaluated on such an orbit becomes a function on the projective Hilbert space $\mathbf{C P}^{N-1}$. This is the case we are most interested in, so we define

$$
f_{H}=\frac{N^{2}}{2} \sum_{i, j \neq 0,0}\left(\left|\left\langle\psi_{0} \mid \psi_{i j}\right\rangle\right|^{2}-\frac{1}{N+1}\right)^{2} .
$$

Because of group covariance there are only $N^{2}-1$ terms in the sum.

The definition of $f_{H}$ can be manipulated further $[4,13]$. Following Appleby, Dang and Fuchs we use an explicit matrix representation of $D_{i j}$ to write 


$$
\left\langle\psi_{0}\left|D_{i j}\right| \psi_{0}\right\rangle=\bar{Z}_{a} q^{b j} \delta_{b+i}^{a} Z^{b}=q^{(a-b) j} \bar{Z}_{a} Z_{a-i} .
$$

Then we take the Fourier transform

$$
\frac{1}{N} \sum_{j} q^{k j}\left|\left\langle\psi_{0}\left|D_{i j}\right| \psi_{0}\right\rangle\right|^{2}=\sum_{a} \bar{Z}_{a} \bar{Z}_{a+k-i} Z_{a+k} Z_{a-i} .
$$

From this it is easy to show that the first frame potential $F_{1}=N^{3}$ (this is true for all SIC-POVMs), and moreover that

$$
f_{H}=\frac{N^{3}}{2}\left(\sum_{i=0}^{N-1} \sum_{k=0}^{N-1}\left|\sum_{a=0}^{N-1} \bar{Z}_{a} \bar{Z}_{a+k-i} Z_{a+k} Z_{a-i}\right|^{2}-\frac{2}{N+1}\right) .
$$

This is the expression we will work with in the sequel.

We will be interested in averages of $f$ and $f_{H}$. To compute these averages we use the Fubini-Study measure $\mathrm{d} \mu_{\mathrm{FS}}$ on projective Hilbert space [14]; this is the natural definition of an average in the absence of any special information. What we wish to compute is

$$
\langle f\rangle \equiv \frac{1}{\operatorname{vol}\left[\mathbf{C P}^{N-1}\right]} \int \mathrm{d} \mu_{\mathrm{FS}} f,
$$

and similarly for $f_{H}$. Computing $\langle f\rangle$ is straightforward: using first the linearity of the expectation value and then the unitary invariance of the measure we obtain

$$
\langle f\rangle=\frac{N^{2}\left(N^{2}-1\right)}{2}\left\langle\left(\left|\left\langle\psi_{0} \mid \psi\right\rangle\right|^{2}-\frac{1}{N+1}\right)^{2}\right\rangle .
$$

Here $\left|\psi_{0}\right\rangle$ is any fixed vector. To compute $\left\langle f_{H}\right\rangle$ requires more work - we will fall back on the explicit expression (13), and then collect terms.

We have now defined the functions we wish to average, and we have defined "average". It remains to define the special subspaces of Hilbert space that we are about to consider.

\section{Special subspaces}


We will compute averages of $f_{H}$ also when the fiducial vector is confined to lie in certain interesting subspaces of the Hilbert space, picked out by the Clifford group. By definition the latter is the normalizer of the HeisenbergWeyl group in the group of all unitaries. Thus the Clifford group is the group of all unitaries $U$ such that

$$
U D_{i j} U^{\dagger}=\omega D_{i^{\prime} j^{\prime}}
$$

where $\omega$ is a phase factor. We are interested in representations up to a phase, and it can be shown that the Clifford group with the phases ignored is isomorphic to a semi-direct product of a symplectic group with the Heisenberg group itself. The importance of this automorphism group was stressed by Zauner [1] and Grassl [8]; for a self-contained account see Appleby [9].

When the dimension is odd the Clifford group contains elements of order 2. They play a major role in the definition of discrete Wigner functions for the odd dimensional case $[15,16]$, and for the special case when the dimension is an odd prime number they are also known as Wootters' phase point operators [17]. They are symmetries of an elliptic curve associated to a complete set of mutually unbiased bases [18]. An elliptic curve can be defined as an embedding of a torus into complex projective space. The Heisenberg group acts on this torus, and gives rise to $N^{2}$ torsion points on the curve, roughly analogous to the $N$ th roots of unity on a circle. Hughston [19] has made the interesting comment that, in the special case $N=3$, these torsion points define a SIC. In general it is known that the torsion points lie in the $N^{2}$ subspaces defined by elements of the Clifford group of order 2. In particular there is such an element $A$ obeying

$$
A D_{i j} A=D_{-i,-j} .
$$

Because $A^{2}=1$, the operator $A$ is both unitary and Hermitean. It splits Hilbert space into two subspaces $\mathcal{H}^{ \pm}$of dimensions $n$ and $n-1$, respectively, where $N=2 n-1$. Explicitly these subspaces are defined by 


$$
\mathcal{H}^{+}: \frac{1}{\sqrt{2}}\left(\begin{array}{c}
\sqrt{2} x_{0} \\
x_{1} \\
\vdots \\
x_{n-1} \\
x_{n-1} \\
\vdots \\
x_{1}
\end{array}\right) \quad \mathcal{H}^{-}: \frac{1}{\sqrt{2}}\left(\begin{array}{c}
0 \\
x_{1} \\
\vdots \\
x_{n-1} \\
-x_{n-1} \\
\vdots \\
-x_{1}
\end{array}\right) .
$$

Unfortunately the fiducial vectors of the SICs do not lie in these subspaces when $N>3$. However, we believe that $f_{H}$ averaged using the Fubini-Study measure in such subspaces gives some feeling for the systematics of the SIC problem, and we will compute this average.

For any $N$ the Clifford group contains elements of order 3. Zauner [1] conjectured that there always exists a SIC such that the fiducial vector is an eigenvector of a symplectic transformation of order 3 , and his conjecture has been verified by Appleby [9] in all available cases [2]. We would therefore like to know the average of $f_{H}$ over such subspaces. Unfortunately it is not so easy to describe these subspaces for arbitrary $N$, and we therefore confined ourselves to the special case $N=7$. Then there exists an element of order 3 acting like a permutation matrix, and the subspaces are explicitly

$$
\mathcal{H}_{1}: \frac{1}{\sqrt{3}}\left(\begin{array}{c}
\sqrt{3} x_{0} \\
x_{1} \\
x_{1} \\
x_{2} \\
x_{1} \\
x_{2} \\
x_{2}
\end{array}\right) \quad \mathcal{H}_{\alpha}: \frac{1}{\sqrt{3}}\left(\begin{array}{c}
0 \\
x_{1} \\
\alpha^{2} x_{1} \\
x_{2} \\
\alpha x_{1} \\
\alpha x_{2} \\
\alpha^{2} x_{2}
\end{array}\right)
$$

where $\alpha$, the eigenvalue, is a primitive third root of unity. The dimension of the subspace $\mathcal{H}_{1}$ is 3 , and the dimensions of the two orthogonal subspaces are 2. There is a fiducial vector for a SIC in $\mathcal{H}_{1}$ [9]. It seemed reasonable to expect that the average $f_{H}$ over this subspace would be quite low, but this expectation was not borne out.

\section{Calculations}


In order to average the frame potential (13) over the entire Hilbert space, we observe - see eq. (25) below - that the angular integrals will make all non-real terms of the sum disappear. We also note that a term of the form $\left|Z_{1}\right|^{4}\left|Z_{2}\right|^{4}$ gives the exact same contribution to the average as one of, say, the form $\left|Z_{3}\right|^{4}\left|Z_{5}\right|^{4}$, and all one needs to keep track of is the number of different $\left|Z_{i}\right|$ in a term, as well as the exponents present. Consequently, we look at the sum (13), and ask in how many ways we can get a term of the type $\left|Z_{1}\right|^{8}$, in how many ways one of the type $\left|Z_{1}\right|^{6}\left|Z_{2}\right|^{2}$, and so on. The problem has then been reduced to elementary equation solving.

Computing the average of $f_{H}$ as given in (13), amounts to computing the average

$$
\langle\Sigma\rangle=\left\langle\sum_{i=0}^{N-1} \sum_{k=0}^{N-1}\left|\sum_{a=0}^{N-1} \bar{Z}_{a} \bar{Z}_{a+k-i} Z_{a+k} Z_{a-i}\right|^{2}\right\rangle .
$$

For calculational convenience, the sum can be split into two parts: the $i=0$ part where all terms are real and we have to keep track of cross terms, and the remaining $i \neq 0$ part, where cross terms contribute only in special cases:

$$
\langle\Sigma\rangle=\sum_{k=0}^{N-1}\left(\sum_{a=0}^{N-1}\left|Z_{a}\right|^{2}\left|Z_{a+k}\right|^{2}\right)^{2}+\sum_{i=1}^{N-1} \sum_{k=0}^{N-1}\left|\sum_{a=0}^{N-1} \bar{Z}_{a} \bar{Z}_{a+k-i} Z_{a+k} Z_{a-i}\right|^{2} .
$$

Let us first consider the $i=0$ term, which can be rewritten as

$$
\sum_{k=0}^{N-1} \sum_{a=0}^{N-1} \sum_{b=0}^{N-1}\left|Z_{a}\right|^{2}\left|Z_{a+k}\right|^{2}\left|Z_{b}\right|^{2}\left|Z_{b+k}\right|^{2}
$$

We will divide our analysis into two cases, the case $a=b$ and the case $a \neq b$. When $a=b$ the terms we get will be either of the type $\left|Z_{1}\right|^{8}$ - this will occur for $k=0$ - or of the type $\left|Z_{1}\right|^{4}\left|Z_{2}\right|^{4}$, which will be the case for all remaining values of $k$, no matter what is the value of $a$ and $b$. The total contribution, modulo integration, from the $i=0$ term will for $a=b$ then be

$$
N\left|Z_{1}\right|^{8}+N(N-1)\left|Z_{1}\right|^{4}\left|Z_{2}\right|^{4},
$$

where the factor $N$ in front of the first term comes from the number of possible choices of $a=b$, and the second term is multiplied by the factor $N(N-1)$ for $N$ different choices of $a=b$ and $N-1$ choices of $k \neq 0$. 
We now turn to the sum for different values of $a$ and $b$. In this case, we will have four different types of $\left|Z_{i}\right|$, each to the power of two, in each term, except in the three special cases where the values of two or more of the indices coincide, namely when $a=b+k, b=a+k$ and $k=0$. In the first two cases we get terms of the $\left|Z_{1}\right|^{4}\left|Z_{2}\right|^{2}\left|Z_{3}\right|^{2}$ type, in the last we get a $\left|Z_{1}\right|^{4}\left|Z_{2}\right|^{4}$ type term. The remaining choices of $k, a$ and $b$-there are $N(N-1)(N-3)$ of them-give terms with all indices different, i.e. terms of the type $\left|Z_{1}\right|^{2}\left|Z_{2}\right|^{2}\left|Z_{3}\right|^{2}\left|Z_{4}\right|^{2}$. The total contribution from the $i=0$ term is given in table 1 .

Moving on to the $i \neq 0$ case, we can forget about cross terms except when $k=0$. Consequently, when $k \neq 0$ we can consider the sum

$$
\sum_{i=1}^{N-1} \sum_{k=1}^{N-1} \sum_{a=0}^{N-1}\left|Z_{a}\right|^{2}\left|Z_{a+k-i}\right|^{2}\left|Z_{a+k}\right|^{2}\left|Z_{a-i}\right|^{2},
$$

while when $k=0$ we still have to work with the expression from equation (21). As in the previous case, we ask what special cases occur, i.e. when two or more indices take on equal values. These cases turn out to be $k=i$, $k=-i$ and $k=0$. The respective contributions to the average are given in the table, as is the contribution for all other choices of indices.

\begin{tabular}{|l|c|c|c|c|c|}
\hline & $Z_{1}^{8}$ & $Z_{1}^{6} Z_{2}^{2}$ & $Z_{1}^{4} Z_{2}^{4}$ & $Z_{1}^{4} Z_{2}^{2} Z_{3}^{2}$ & $Z_{1}^{2} Z_{2}^{2} Z_{3}^{2} Z_{4}^{2}$ \\
\hline$i=0, a=b$ & $N$ & - & $N(N-1)$ & - & - \\
\hline$i=0, a \neq b, k=0$ & - & - & $N(N-1)$ & - & - \\
\hline$i=0, a \neq b, k=a-b$ & - & - & - & $N(N-1)$ & - \\
\hline$i=0, a \neq b, k=b-a$ & - & - & - & $N(N-1)$ & - \\
\hline$i=0$, others & - & - & - & - & $N(N-1)(N-3)$ \\
\hline$i \neq 0, k=0$ & - & - & $N(N-1)$ & $2 N(N-1)$ & $N(N-1)(N-3)$ \\
\hline$i \neq 0, k=i$ & - & - & - & $N(N-1)$ & - \\
\hline$i \neq 0, k=-i$ & - & - & - & $N(N-1)$ & - \\
\hline$i \neq 0$, others & - & - & - & - & $N(N-1)(N-3)$ \\
\hline total & $N$ & - & $3 N(N-1)$ & $6 N(N-1)$ & $3 N(N-1)(N-3)$ \\
\hline
\end{tabular}

Table 1: Number of different type terms in the average for odd $N$.

Subtracting the constant term and multiplying by the initial factor of (13) yields an expression which can be integrated over all of Hilbert space 
in order to obtain the SIC-function average. To perform the integrals we parametrize the unit vectors as

$$
Z^{a}=\left(\sqrt{p_{0}}, \sqrt{p_{k}} e^{i \nu_{k}}\right)
$$

where the ranges of the parameters are

$$
p_{0}+p_{1}+\ldots+p_{N-1}=1, \quad p_{0} \geq 0, \quad p_{k} \geq 0, \quad 0 \leq \nu_{k}<2 \pi .
$$

We solve for $p_{0}$, and obtain the explicit expression [14]

$$
\langle f\rangle=\frac{(N-1) !}{(2 \pi)^{N-1}} \int_{0}^{1} \mathrm{~d} p_{1} \ldots \int_{0}^{1-p_{1}-\ldots-p_{N-2}} \mathrm{dp}_{N-1} \int_{0}^{2 \pi} \mathrm{d} \nu_{1} \ldots \int_{0}^{2 \pi} \mathrm{d} \nu_{N-1} f
$$

In calculating the averages a number of standard integrals will be used, namely

$$
\begin{gathered}
\left\langle\left|Z_{1} Z_{2} Z_{3} Z_{4}\right|^{2}\right\rangle=\frac{(N-1) !}{(N+3) !} \\
\left\langle\left|Z_{1}\right|^{4}\left|Z_{2} Z_{3}\right|^{2}\right\rangle=2 \frac{(N-1) !}{(N+3) !} \\
\left\langle\left|Z_{1} Z_{2}\right|^{4}\right\rangle=2^{2} \frac{(N-1) !}{(N+3) !} \\
\left\langle\left|Z_{1}\right|^{6}\left|Z_{2}\right|^{2}\right\rangle=3 ! \frac{(N-1) !}{(N+3) !} \\
\left\langle\left|Z_{1}\right|^{8}\right\rangle=4 ! \frac{(N-1) !}{(N+3) !}
\end{gathered}
$$

The computations for odd values of $N$ are straightforward, with few complications. Even $N$ are, however, a somewhat different story. To begin with, cross terms play in also for non-zero values of $i$ and $k$, and in a somewhat different manner than in the odd $N$ case. Whereas we get cross term contributions for odd $N$ only when all terms are real in a sum-as for $k=0$, for 
instance - we here find pairwise equal terms inside the modulus sign of the $i \neq 0$ term of equation (21), so that care has to be exercised when squaring. Using the same strategy as for odd $N$-dividing the sum into $i=0$ and $i \neq 0$ parts and asking when two or more indices are equal - we find a number of index choices for which terms of order higher than two occur, some of which coincide with the ones for odd $N$. As opposed to in the odd $N$ case, though, the number of possible choices for the summation indices depend on the parity of $i$, as well as on the modulo four value of $N$, so that the calculational details differ beteween, for instance, $N=6$ and $N=8$. This is because the restrictions one gets when solving the equal-indices-equations, are sometimes equivalent for, say, $i=3$ and $N=0 \bmod 4$ and independent for $N=2 \bmod$ 4 for the same $i$, giving a different number of combinatorial possibilities. The final results for the frame potential average taken over Hilbert space, on the other hand, turns out to be identical for the two cases; this average is given in section 5 .

Also when considering the sum average over $\mathcal{H}^{+}$and $\mathcal{H}^{-}$, the calculations get more involved than in the non-restricted odd $N$ case. As for even $N$, we get cross term contributions to the integral, and further technical complications are due to the fact that the respective subspace conditions,

$$
Z_{i}=Z_{-i}
$$

and

$$
Z_{i}=-Z_{-i}
$$

make the number of equations to solve when asking what possibilities there are for the power of any $Z_{i}$ to be higher than two in a term larger. In other words, the calculations have to be divided into a greater number of different cases, but the logic is much the same as in the calculations for all of Hilbert space sketched above. In using equations (26 - 30), one should keep in mind that the dimension of the space over which the average is taken is no longer equal to $N$.

\section{Results}

Our results are easy to state. The function $f$ attains its global minimum at a SIC, and its global maximum if all the $N^{2}$ unit vectors coincide. Thus 


$$
0 \leq f \leq \frac{N^{4}}{2} \frac{N-1}{N+1}
$$

Its Fubini-Study average value is

$$
\langle f\rangle_{\mathrm{FS}}=\frac{N^{2}}{2} \frac{N-1}{N+1} .
$$

When the dimension is large, the scalar product between randomly choosen vectors is close to zero, and this is reflected by the average.

If we specialize to $N^{2}$ unit vectors forming an orbit under the WeylHeisenberg group we believe that the global maximum occurs if the fiducial vector is an eigenvector of some element in the group, in which case the orbit collapses to an eigenbasis. Thus

$$
0 \leq f_{H} \leq \frac{N^{3}}{2} \frac{N-1}{N+1}
$$

(Unfortunately we were unable to prove that the eigenbasis represents the global maximum. It seems obvious that this is so, and we did check that it is a local extremum. For safety, we do not number this equation.) The average value depends on whether the Hilbert space dimension is odd or even:

$$
\begin{gathered}
N=2 n-1: \quad\left\langle f_{H}\right\rangle_{\mathrm{FS}}=\frac{N^{2}}{2} \frac{N(N-1)}{(N+2)(N+1)} \\
N=2 n: \quad\left\langle f_{H}\right\rangle_{\mathrm{FS}}=\frac{N^{2}}{2} \frac{N^{2}}{(N+3)(N+1)} .
\end{gathered}
$$

Asymptotically there is no difference. It is perhaps somewhat unexpected that the average of $f_{H}$ has the same asymptotic behaviour as the average of $f$.

We also computed the average when the fiducial vector is restricted to lie in certain interesting subspaces defined by elements of the Clifford group (the normalizer of the Weyl-Heisenberg group). Elements of order 2 occur in odd dimensions $N=2 n-1$. Their eigenspaces have dimension $\operatorname{dim}\left[\mathcal{H}^{-}\right]=n-1$ and $\operatorname{dim}\left[\mathcal{H}^{+}\right]=n$. As observed by Hughston [19], when $N=3 \mathcal{H}^{-}$contains only one vector, and it is a fiducial vector for a SIC. However, 3 is a very special dimension. The complementary subspace $\mathcal{H}^{+}$contains a SIC fiducial 
vector too, as well as four vectors from a complete set of four mutually unbiased bases, for which $f_{H}$ attains its (conjectured) maximum. The average over $\mathcal{H}^{+}$is $\left\langle f_{H}\right\rangle=81 / 40$. For $N>3$ the situation is quite different. We find

$$
N=2 n-1>3: \quad\left\langle f_{H}\right\rangle_{\mathcal{H}^{-}}=\left\langle f_{H}\right\rangle_{\mathcal{H}^{+}}=N^{2} \frac{N(N-1)}{(N+3)(N+1)} .
$$

Asymptotically this is twice the average over the full Hilbert space. It is a bit striking that the averages are equal.

It is harder to deal with the subspaces defined by elements of order 3 . At the same time they are more interesting, because Zauner's conjecture $[1,9,20]$ says that the fiducial vector can always be choosen to lie in the subspace with eigenvalue 1 . We did compute averages for $N=7$. There are three subspaces $\mathcal{H}_{1}, \mathcal{H}_{\alpha}, \mathcal{H}_{\alpha^{2}}$, labelled by the eigenvalues of the element that cubes to one $\left(\alpha=e^{2 \pi i / 3}\right)$. Their dimensions are respectively 3,2 , and 2 . The average values of $f_{H}$ in the three subspaces are

$$
\begin{gathered}
\left\langle f_{H}\right\rangle_{\mathcal{H}_{1}}=\frac{151 \cdot 7^{3}}{5 \cdot 3^{4} \cdot 2^{3}} \approx 15.985 \\
\left\langle f_{H}\right\rangle_{\mathcal{H}_{\alpha}}=\left\langle f_{H}\right\rangle_{\mathcal{H}_{\alpha^{2}}}=\frac{37 \cdot 7^{3}}{5 \cdot 3^{3} \cdot 2^{3}} \approx 11.751 .
\end{gathered}
$$

The average over the subspace that contains the SIC fiducial vector is above the average $\left\langle f_{H}\right\rangle$ over the full Hilbert space. This surprised us.

The values of $f_{H}$ vary widely, also within the special subspaces (except that $f_{H}$ is actually constant on $\mathcal{H}^{-}$when $N=5$ ). We describe the situation for $N=7$ in the following table:

\begin{tabular}{|l||c|c|c|c|c|c|}
\hline & $f$ & $f_{H}$ & $f_{H}\left(\mathcal{H}^{+}\right)$ & $f_{H}\left(\mathcal{H}^{-}\right)$ & $f_{H}\left(\mathcal{H}_{1}\right)$ & $f_{H}\left(\mathcal{H}_{\alpha}\right)$ \\
\hline Minimum & 0 & 0 & $12.2(?)$ & $4.764(?)$ & 0 & $?$ \\
Average & 18.375 & 14.29 & 25.72 & 25.72 & 15.98 & 11.75 \\
Maximum & 900.4 & $128.6(?)$ & $128.6(?)$ & $42.88(?)$ & $?$ & $?$ \\
\hline
\end{tabular}

Some entries were left uncomputed, and some without proofs - we marked the latter with a question mark within bracket, even though we are sure they are correct. 
Unfortunately we are unable to see how to compute the asymptotic behaviour of $\left\langle f_{H}\right\rangle$ for a fiducial vector in subspaces of the kind considered by Zauner. We expect them to depend on number theoretical details of the dimension. It would be interesting to see numerical studies of such averages however.

\section{References}

[1] G. Zauner: Quantendesigns. Grundzüge einer nichtkommutativen Designtheorie, PhD thesis, Univ. Wien 1999.

[2] J. M. Renes, R. Blume-Kohout, A. J. Scott, and C. M. Caves, Symmetric informationally complete quantum measurements, J. Math. Phys. 45 (2004) 2171.

[3] A. J. Scott, Tight informationally complete quantum measurements, J. Phys. A39 (2006) 13507.

[4] D. M. Appleby, H. B. Dang, and C. A. Fuchs, Physical significance of symmetric informationally-complete sets of quantum states, arXiv:0806.1339.

[5] P. W. H. Lemmens and J. J. Seidel, Equiangular lines, J. Algebra 24 (1973) 494.

[6] J. J. Benedetto and M. Fickus, Finite normalized tight frames, Adv. Comp. Math. 18 (2003) 357.

[7] T. Strohmer and R. W. Heath Jr., Grassmannian frames with applications to coding and communication, Appl. Comput. Harmon. Anal. 14 (2003) 257.

[8] M. Grassl, On SIC-POVMs and MUBs in dimension 6, in Proc. of ERATO conference on Quantum Information Science (2004), Tokyo 2004.

[9] D. M. Appleby, SIC-POVMs and the extended Clifford group, J. Math. Phys. 46, 052107 (2005). 
[10] M. Grassl, Tomography of quantum states in small dimensions, Electronic Notes in Discrete Math. 20 (2005) 151.

[11] M. Grassl, Finding equiangular lines in complex space, talk at the Magma workshop, 2006.

[12] H. Weyl: Theory of Groups and Quantum Mechanics, Dutton, New York 1932.

[13] M. Khatirinejad, On Weyl-Heisenberg orbits of equiangular lines, J. Algebr. Comb., published online 6 November 2007.

[14] I. Bengtsson and K. Życzkowski: Geometry of Quantum States, Cambridge UP 2006.

[15] S. Chaturvedi, E. Ercolessi, G. Marmo, G. Morandi, N. Mukunda and R. Simon, Wigner distributions for finite dimensional quantum systems: An algebraic approach, Pramana J. Phys. 65 (2005) 981.

[16] D. Gross, Hudson's theorem for finite-dimensional quantum systems, J. Math. Phys. 47 (2006) 122107.

[17] W. K. Wootters, A Wigner-function formulation of finite-state quantum mechanics, Ann. Phys. 176 (1987) 1.

[18] K. Hulek, Projective geometry of elliptic curves, Asterisque 137 (1986) 1.

[19] L. P. Hughston, unpublished.

[20] S. T. Flammia, On SIC-POVMs in prime dimensions, J. Phys. A39 (2006) 13483. 